\title{
Comparison of the Attitudes of Nurses and Physicians toward Palliative Care in Neonatal Intensive Care Units
}

\author{
Ha Na Jung, R.N. and Hyeon Ok Ju, Ph.D.* \\ Department of Nursing, Pusan National University Hospital, *Department of Nursing, Dong-A University, Busan, Korea
}

Purpose: This study aimed to compare the attitudes of nurses and physicians toward neonatal palliative care and identify the barriers to and facilitators of neonatal palliative care, with the goal of improving palliative care for infants in neonatal intensive care units (NICUs). Methods: This cross-sectional study analyzed data from the NICUs of seven general hospitals with 112 nurses and 52 physicians participating. Data were collected using the $\mathrm{Neo}^{-}$ natal Palliative Care Attitude Scale questionnaire. Results: Only 12.5\% of nurses and 11.5\% of physicians reported that they had sufficient education in neonatal palliative care. In contrast, $89.3 \%$ of the nurses and $84.6 \%$ of the physicians reported that they needed further education. The common facilitators for both nurses and physicians were: 1) agreement by all members of the department regarding the provision of palliative care and 2) informing parents about palliative care options. The common barriers for both nurses and physicians were: 1) policies or guidelines supporting palliative care were not available, 2) counseling was not available, 3) technological imperatives, and 4) parental demands for continuing life support. Insufficient resources, staff, and time were also identified as barriers for nurses, whereas these were not identified as barriers for physicians. Conclusion: It is necessary to develop hospital or national guidelines and educational programs on neonatal palliative care, and it is equally necessary to spread social awareness of the importance of neonatal palliative care.

Key Words: Neonatal intensive care, Palliative care, Attitude
Received June 25, 2021

Revised July 29, 2021

Accepted August 4, 2021

\author{
Correspondence to \\ Hyeon Ok Ju \\ ORCID: \\ https://orcid.org/0000-0002-0264-2651 \\ E-mail: enfanju@dau.ac.kr \\ The article is a revision of the first \\ author's master's thesis from Dong-A \\ University.
}

\section{INTRODUCTION}

Neonatal palliative care is defined as the provision of holistic and extensive care for babies who are not expected to survive [1]. Once a diagnosis has been made with a low probability of survival, palliative care can provide babies with comfort instead of more aggressive procedures that cause pain and distress, such as ventilator treatment and cardiopulmonary resuscitation (CPR) [2]. Parents and family members are also provided with psychological, mental, and social support [3].

Although the number of births in Korea is steadily decreas- ing (470,200 in 2010 and 272,400 in 2020) [4], the increase in older mothers has led to an increased incidence of high-risk neonates who need to be hospitalized in a NICU for tests and treatments (5.7\% in 2009 and 8.1\% in 2019) [5,6]. Despite a low probability of survival, high-risk neonates often receive invasive and intensive treatments such as tracheal intubation, peripheral and central venous catheter insertion, ventilator treatment, and CPR [7] immediately after birth. Such treatments can cause great pain in neonates [8] and exacerbate neurological defects, as well as prolong the process of death [9]. Previous research found that babies who received neonatal 
palliative care instead of invasive and intensive treatments underwent fewer painful procedures, such as CPR. Their parents, receiving psychological and social support, were more satisfied with the treatment process [3]. In addition, the medical staff could provide the babies with necessary treatments while also respecting the dignity and best interests of the babies [10]. $\mathrm{Be}^{-}$ cause of these advantages, there is a growing call for neonatal palliative care options [11]. However, the concept of neonatal palliative care is still not widespread [10] and has received little attention [2], partly due to the fundamental societal belief that babies should not die [12]. In addition, medical staff are sometimes blamed for the death of a baby because of treatments performed (or not performed) while under their care [13].

To improve negative perceptions of neonatal palliative care and promote its practice, it is essential that medical staff work together and share similar objectives [14]. Collaboration between nurses (who spend the most time with patients) [15] and physicians (who make decisions regarding palliative care through discussions with families) can lead to a successful practice of neonatal palliative care [16]. A positive attitude toward palliative care by the medical staff may help satisfy the demands of patients and families and can serve as a facilitator of palliative care [17]. In contrast, a negative attitude can be a barrier to the practice of palliative care [18]. Therefore, to provide neonatal palliative care effectively, it is important to study the facilitators of and barriers to palliative care practice [19], understand the attitudes of nurses and physicians toward neonatal palliative care, and analyze the differences between the two groups in advance.

Kain et al. [20] defined a facilitator as something that can assist in bringing about an outcome by providing indirect or unobtrusive assistance, guidance, or supervision, and a barrier as any obstruction that may impede the adoption of a palliative model of care in neonatal nursing. To measure the facilitators of and barriers to neonatal palliative care, Kain et al. [20] developed a palliative care attitude scale, and studies were conducted in Iran [21], the United States [19], and Taiwan [8] with this instrument. Although there have been studies to promote neonatal palliative care overseas, in South Korea, palliative care has been focused mainly on adults [22] and there have been few studies on neonatal palliative care.
Therefore, this study aims to increase awareness of neonatal palliative care by investigating the attitudes of nurses and physicians in the NICU toward neonatal palliative care, comparing the differences between both groups, and by identifying the facilitators of and barriers to neonatal palliative care. This study also aims to provide basic data that may help identify measures that diminish the barriers to palliative care.

\section{METHODS}

\section{Study design}

This is a descriptive study designed to investigate and compare the attitudes of nurses and physicians in the NICU toward neonatal palliative care, as well as to identify the facilitators of and barriers to neonatal palliative care.

\section{Participants}

The participants in this study were nurses and physicians working in the NICU at seven general hospitals located in B City and G Province who understood the purpose of the study and agreed to participate. The inclusion criteria for participation in the study were nurses who provided direct care to high-risk neonates and had worked in the NICU for a year or longer and physicians, pediatric residents, or specialists who currently worked in the NICU. The study samples (questionnaires) totaled 144 (96 from nurses and 48 from physicians). The sample size was calculated using the $G^{*}$ Power 3.1.9.2 program, with the independent $\mathrm{t}$-test, a significance level of 0.05 , a medium effect size of 0.50 , a statistical power of 0.80 , and a nurse-to-physician ratio of 2:1. Anticipating a dropout rate of $20 \%$, questionnaires were distributed to a total of 179 participants (123 nurses and 56 physicians). A total of 164 questionnaires were used for the final analysis, excluding those with incomplete or inappropriate answers (11 from nurses and 4 from physicians). The drop-out rate was $9 \%$ for nurses and $7 \%$ for physicians.

\section{Study tools}

\section{1) General characteristics of the participants and characteristics of their attitudes toward palliative care}

The general characteristics of the participants included 7 
items: gender, age, marital status, religion, position at work, highest level of education, and work experience in the NICU. The characteristics of their attitudes toward palliative care included 3 items: educational experience, educational needs, and experience in neonatal palliative care.

\section{2) Attitudes toward neonatal palliative care}

The Neonatal Palliative Care Attitude Scale (NPCAS) developed by Kain et al. [20] was translated into Korean, and the reliability and content validity were reviewed before it was used in this study. Permission to use the tool and to translate it from English into Korean was obtained from the author via email. A bilingual researcher who was familiar with the terminology of the instrument as well as the neonatal palliative care environment translated the original tool into Korean as a rough draft. A literal translation was avoided so that it could be easily read and understood by the nurses and physicians. Preliminary surveys were completed by two nurses with over 10 years of experience in the NICU and four pediatric residents to verify the legibility and cultural validity of the tool. The phrase "dying baby," a literal translation in the original tool, was changed to "neonates near death." Afterwards, the validity of its content was reviewed by one professor of nursing, four NICU nurses, and two pediatric physicians, and the resulting content validity index of all items was 0.80 or higher. An expert fluent in both English and Korean back-translated the tool, and a native speaker verified the correspondence of the original and translated tools.

The instrument comprises a total of 12 items in three categories: organization (five items), resources (five items), and clinician (two items). Items in the "organization" category relate to the environment of the NICU and whether they promote or interfere with the practice of palliative care. Items in the "resources" category include various human and material resources in the NICU. Items in the "clinician" category deal with the moral and ethical concerns of the medical staff. Each item is assessed using the 5-point Likert scale from 1 (strongly disagree) to 5 (strongly agree). Kain et al. [20] interpreted a score of 3.0 or higher in the "organization" and "resources" categories to be indicative of facilitators and a score of lower than 3.0 to reflect barriers, while the interpretation was reversed in the "clinician" category. However, the total score was not used. Although the original tool is a 5-point Likert scale, tool development research and studies from other countries that applied this tool classified facilitators and barriers by scores, while specific analyses were conducted using the proportions of responses to each item. To facilitate comparisons with previous research, the answers to each question were classified as "agree," "unsure," and "disagree," and the mean scores were provided. Cronbach's alpha in the study of Kain et al. [20] was 0.73 for "organization," 0.65 for "resources," and 0.63 for "clinician," and the corresponding values were 0.63 , 0.76 , and 0.64 , respectively, in this study.

\section{Data collection}

The data were collected from September 19, 2019 to October 21, 2019. After Institutional Review Board (IRB) approval from D University and permission and cooperation from the department of nursing and medical office were obtained, a recruitment notice was posted in the NICU and the medical office of the pediatrics department at each hospital. After being contacted by participants who expressed interest in participating in the study, the researcher visited the NICU and the medical office of the relevant hospitals. Questionnaires were distributed per the study protocol and were explained to the participants. Informed consent forms were received. The researcher retrieved the sealed questionnaires in person a week later.

\section{Data analysis}

The collected data were analyzed using SPSS version 25.0 (IBM Corp., Armonk, NY, USA). The general characteristics of the nurses and physicians and the characteristics of their attitudes toward palliative care were analyzed by frequency and percentage. Differences between the two groups were analyzed using the chi-square test, and their attitudes toward palliative care were assessed in terms frequency, percentage, mean, and standard deviation. Differences between the nurses' and physicians' attitudes toward palliative care were analyzed using the independent $\mathrm{t}$-test.

\section{Ethical considerations}

To help secure the ethical integrity of this research prior to conducting the study, approval was obtained from the IRB of 
D University (2-104709-AB-N-01-201908-BR-014-02). Before the study participants filled out a questionnaire, the purpose and process of the study were explained to them by the investigator and written consent was obtained. The explanatory statement confirmed that all personal information would remain confidential, that the participants could discontinue the study at any time if they no longer wished to participate, and that the study data would not be used for purposes other than research. The statement also notified participants that all electronic documents would be deleted permanently and that all paper documents would be shredded 3 years after the study was completed.

\section{RESULTS}

\section{General characteristics of the participants and characteristics of their attitudes regarding palliative care}

A total of 164 participants (112 nurses and 52 physicians) participated in this study, and the general characteristics of those participants and their attitudes toward palliative care are shown in Table 1. The nurses were all female, while $32.7 \%$ of the physicians were male and $67.3 \%$ were female $(\mathrm{P}<0.001)$. Most (67.9\%) of the nurses were in their 20s, while $59.6 \%$ of the physicians were in their $30 \mathrm{~s}(\mathrm{P}<0.001)$. The data revealed differences in marital status, as $83.0 \%$ of the nurses were unmarried and $53.8 \%$ of the physicians were married $(\mathrm{P}<0.001)$. The majority of both nurses and physicians $(87.5 \%$ and $88.5 \%$, respectively) had not received education in neonatal palliative care $(\mathrm{P}<0.861)$, and $89.3 \%$ of the nurses and $84.6 \%$ of the physicians answered that education on neonatal palliative care was necessary $(P<0.395)$. There was no significant difference between the two groups in the proportion of participants who had provided neonatal palliative care: $29.5 \%$ of the nurses and $21.2 \%$ of the physicians $(\mathrm{P}<0.264)$.

\section{Attitudes of participants toward palliative care}

The attitudes of the nurses and physicians toward palliative care are shown in Table 2.

In the organization category, "parents are involved in decision-making” was scored highest by both nurses (4.17 [ \pm
Table 1. General Characteristics of Nurses and Physicians ( $N=164)$.

\begin{tabular}{|c|c|c|c|c|}
\hline \multirow[t]{2}{*}{ Variables } & \multirow{2}{*}{ Categories } & \multirow{2}{*}{$\begin{array}{c}\begin{array}{c}\text { Nurse } \\
(n=112)\end{array} \\
n(\%)\end{array}$} & \multirow{2}{*}{$\begin{array}{c}\begin{array}{c}\text { Physician } \\
(n=52)\end{array} \\
n(\%)\end{array}$} & \multirow[t]{2}{*}{$\mathrm{P}$} \\
\hline & & & & \\
\hline \multirow[t]{2}{*}{ Gender } & Male & $0(0)$ & $17(32.7)$ & $<0.001$ \\
\hline & Female & $112(100)$ & 35 (67.3) & \\
\hline \multirow[t]{3}{*}{ Age (yr) } & $20 \sim 29$ & 76 (67.9) & $17(32.7)$ & $<0.001$ \\
\hline & 30 39 & $27(24.1)$ & $31(59.6)$ & \\
\hline & $\geq 40$ & $9(8.0)$ & $4(7.7)$ & \\
\hline \multirow[t]{2}{*}{ Marital status } & Yes & $19(17.0)$ & $28(53.8)$ & $<0.001$ \\
\hline & No & $93(83.0)$ & $24(46.2)$ & \\
\hline \multirow[t]{2}{*}{ Religious } & Yes & $35(31.2)$ & $29(55.8)$ & 0.003 \\
\hline & No & 77 (68.8) & $23(44.2)$ & \\
\hline \multirow[t]{4}{*}{ Position } & Staff nurse & 106 (94.6) & - & \\
\hline & $\geq$ Charge nurse & $6(5.4)$ & - & \\
\hline & Resident & - & $42(80.8)$ & \\
\hline & Specialist & - & $10(19.2)$ & \\
\hline \multirow[t]{3}{*}{ Education } & College & $16(14.3)$ & - & \\
\hline & University & $83(74.1)$ & - & \\
\hline & $\geq$ Graduate school & $13(11.6)$ & - & \\
\hline \multirow{3}{*}{$\begin{array}{l}\text { Duration: } \\
\text { Years worked in } \\
\text { NICU }\end{array}$} & $1 \sim 4$ & $70(62.5)$ & - & \\
\hline & $5 \sim 9$ & $28(25.0)$ & - & \\
\hline & $\geq 10$ & $14(12.5)$ & - & \\
\hline \multirow{2}{*}{$\begin{array}{l}\text { Education in } \\
\text { neonatal } \\
\text { palliative care }\end{array}$} & Yes & $14(12.5)$ & $6(11.5)$ & 0.861 \\
\hline & No & $98(87.5)$ & 46 (88.5) & \\
\hline \multirow{3}{*}{$\begin{array}{l}\text { Need for neonatal } \\
\text { palliative care } \\
\text { education }\end{array}$} & Yes & $100(89.3)$ & 44 (84.6) & 0.395 \\
\hline & Unsure & $12(10.7)$ & $8(15.4)$ & \\
\hline & No & - & - & \\
\hline \multirow{2}{*}{$\begin{array}{l}\text { Experience } \\
\text { in neonatal } \\
\text { palliative care }\end{array}$} & Yes & $33(29.5)$ & $11(21.2)$ & 0.264 \\
\hline & No & 79 (70.5) & 41 (78.8) & \\
\hline
\end{tabular}

$0.77]$ and physicians (4.12 [ \pm 0.73$]$ ), and those who answered "strongly or somewhat agree" were $84.8 \%$ and $78.8 \%$, respectively. Another item for which a majority of both nurses and physicians answered "agree" was "health care team agrees with and supports palliative care" (59.8\%, 3.62 \pm 0.88 and 55.8\%, $3.50 \pm 0.83$, respectively). In contrast, statistically significant differences between the two groups were found for certain items, where fewer than $50 \%$ of the nurses agreed but more than $50 \%$ of the physicians agreed. These included "medical staff support palliative care" $(46.4 \%, 3.30 \pm 0.92$ and $63.5 \%$, $3.73 \pm 0.63$, respectively; $\mathrm{P}<0.001)$ and "parents are informed of palliative care options" $(46.4 \%, 3.17 \pm 1.11$ and $61.5 \%, 3.54$ \pm 0.98 , respectively; $\mathrm{P}=0.042$ ).

A lower percentage of both nurses and physicians agreed with the item "team can express opinions, values, and beliefs" 


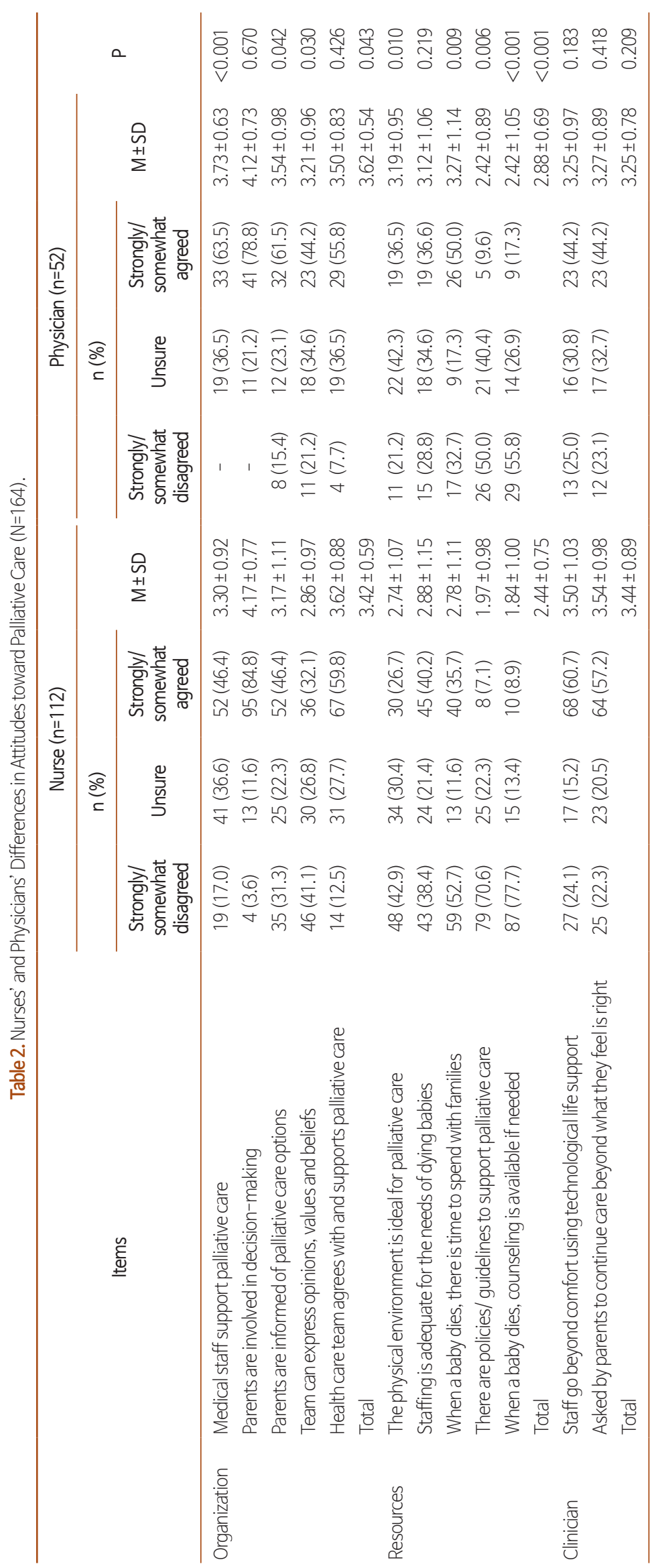


$(32.1 \%, 2.86 \pm 0.97$ and $44.2 \%, 3.21 \pm 0.96$, respectively), and the scores and proportion of agreement for the nurses were significantly lower than those of the physicians $(\mathrm{P}=0.030)$. The overall score was $3.42 \pm 0.59$ for the nurses and $3.62 \pm 0.54$ for the physicians, and the difference was statistically significant $(\mathrm{P}=0.043)$.

All items were defined as a facilitators for the physicians, whereas "team can express opinions, values, and beliefs" was found to be a barrier for the nurses.

In the resources category, $50 \%$ or more of the nurses did not mark "agree" to any item, and no item was scored 3.0 (medium) or higher by the nurses. However, the physicians scored items regarding physical environment, staff, and time at 3.0 or higher. The item with the lowest score for both nurses and physicians was "there are policies/guidelines to support palliative care" $(7.1 \%, 1.97 \pm 0.98$ and $9.6 \%, 2.42 \pm 0.89$, respectively). The overall score was lower than 3.0 in both groups (2.44 \pm 0.75 for nurses and $2.88 \pm 0.69$ for physicians), reflecting a statistically significant difference $(\mathrm{P}<0.001)$.

Although all items in the resources category were barriers for the nurses, only items regarding guidelines and counseling were found to be barriers for the physicians.

In the clinician category, both nurses and physicians had similar scores. The percentages of nurses and physicians who agreed with the item "staff go beyond comfort in using technological life support" were $60.7 \%$ and $44.2 \%$, respectively, and the scores were $3.50 \pm 1.03$ and $3.25 \pm 0.97$, respectively. The percentages of those who agreed with the item "staff are asked by parents to continue care beyond what they feel is right" were $57.2 \%$ and $44.2 \%$, respectively, with scores of 3.54 \pm 0.98 and $3.27 \pm 0.89$, respectively. The overall score for the clinician category was $3.44 \pm 0.89$ for nurses and $3.25 \pm 0.78$ for physicians; this was not a statistically significant difference.

All items in the clinician category were found to be barriers to palliative care for both nurses and physicians.

\section{DISCUSSION}

This study was conducted to increase awareness of neonatal palliative care in nurses and physicians in the NICU by identifying the facilitators of and barriers to palliative care.

The answers from the nurses identified four facilitators and eight barriers, whereas the answers from the physicians identified eight facilitators and four barriers. The items found to be facilitators for both nurses and physicians were "health care team agrees with and supports palliative care," "parents are involved in decision-making," and "parents are informed of palliative care options."

In the study of Kain et al. [20] of 645 NICU nurses in Australia, there were five facilitators (the health care team's support of palliative care; the expression of opinions, values and beliefs; the availability of counseling and guidelines; and the support of parents by the health care team). Kain et al. [20] described the support and agreement of all team members providing palliative care to neonates near death as the key to successful palliative care. Successful palliative care can be accomplished when nurses and physicians work together and all members support palliative care [14]. If nurses and physicians disagree, it may deteriorate the quality of care and bring about negative outcomes [23]. Therefore, it is recommended that the medical staff exchange opinions at regular meetings, reach consensus on differing views, and move forward toward the same objective, which is successful palliative care. Because most physicians and nurses in this study were found to support palliative care, this essential component of the objective can be considered to have been met by this study group.

The study by Wright et al. [19] of 50 NICU nurses in the midwestern United States also reported eight facilitators of palliative care, including parental involvement in decisionmaking and informing parents of palliative care options. Throughout the process of neonatal palliative care, parents go through extreme stress, making numerous choices for their babies [13]. They may experience guilt and have difficulty accepting that their baby will die [24]. Therefore, parents should be given general information on palliative care before they make these important decisions [8]. According to the study by Falck et al. [25] on the mothers of neonates near death, those mothers who were informed about palliative care and able to resolve conflicts and confusion through communication with the medical staff had a higher level of satisfaction with palliative care. Such results suggest that informing parents of palliative care options and their involvement in decisions are essential elements of palliative care practice.

Items found to be barriers by both nurses and physicians 
were: 1) a lack of policies or guidelines to support palliative care, 2) the unavailability of counseling, 3) technological imperatives, and 4) parental demands for continued life support measures. Although policies and guidelines to support palliative care were considered to be facilitators in studies of nurses in the United States and Australia [19,20], they were found to be barriers in studies of nurses in Iran and Taiwan [21,8]. In particular, Chen et al. [8] reported that the reason why the item regarding the adequacy of the physical environment was answered "agree" by almost one half and "disagree" by the other half of respondents was due to the absence of policies and guidelines to support palliative care and emphasized the importance of guidelines. Moreover, according to Wi and Kang [5], the attitudes of nurses who provided palliative care in person toward palliative care itself were more positive when there were protocols or guidelines to support palliative care than when there were not. Thus, to support neonatal palliative care, the development of guidelines or policies should be prioritized.

In the studies of nurses in the United States and Australia [19,20], 68\% and 78\%, respectively, answered that counseling was available if they needed it when a baby died. However, only $8.9 \%$ of the nurses in this study answered that counseling was available. When a baby dies, the medical staff may experience anxiety and trauma as well as feelings of guilt for believing that they failed to do their best [26]. These experiences may cause them to fail to provide appropriate palliative care in other similar situations [20]. However, by sharing their emotions with others, the medical staff can relieve guilty feelings and receive encouragement and comfort [27]. Thus, active mediation for the medical staff, including the consistent provision of counseling and a support system to reduce their stress, trauma, and anxiety, is necessary.

For medical staff, causing discomfort when using technological life support and being asked by parents to continue care beyond what they feel is right caused the same ethical dilemmas as those of other medical professionals in a majority of studies for which the same data collection tool was used $[8,19,28]$. In Australia, $62.2 \%$ of 645 neonatal nurses agreed that they cause discomfort when using technological life support, and $75.7 \%$ of them stated that they were asked by parents to continue care beyond what they feel is right. Ethical dilemmas encountered when caring for end-of-life children have been reported in a number of studies. The medical staff may experience ethical dilemmas and conflicting emotions if they are pressured by parents to continue care in situations where they feel that life-extending care would not be effective $[15,20]$. In addition, the medical staff may end up overtreating to meet parental demands, which may then worsen moral and ethical dilemmas and disrupt the practice of neonatal palliative care [8]. The misconception among parents and medical staff that neonatal palliative care is an abandonment of the baby's life and treatment needs to be corrected. Education of both medical staff and parents is necessary to confirm that palliative care improves the quality-of-life for neonates near death and supports parents and family.

Items identified as barriers in this study were: the expression of opinions, values, and beliefs about palliative care; the physical environment; staffing; and time. When asked if they could express opinions, values, and beliefs about palliative care, more nurses answered that they could not than answered that they could, whereas more physicians answered that they could than could not. This may be because physicians often have a more substantial decision-making role than nurses [2]. Nurses have fewer opportunities to express their opinions when the process of decision-making among medical staff is structured within a hierarchical system.

When analyzing resources, all items-including the physical environment, staffing, time, guidelines, and counseling-were found to be barriers to palliative care for the nurses. Only the lack of guidelines, policies and counseling were found to be barriers for the physicians. In most studies on nurses in the NICU $[8,20,21]$, insufficient staff and time were reported as barriers to palliative care, which corresponds with the results of this study. Although the results of this study cannot be compared with other studies since there is no study on the attitude of physicians toward palliative care using the same tool, this study shows that nurses experienced more barriers related to staffing and time. According to a 2018 survey by the Ministry of Health and Welfare, 3.6 patients were assigned to 1 nurse in the NICU [28]. This is high when compared with Japan where the patient-to-nurse ratio is $3: 1$ and the United States where it is 2:1 [28]. Due to an insufficiency of staff and time, nurses in Korea cannot provide high-quality care to ne- 
onates [29], which is also the case in palliative care. Therefore, medical institutions should address this problem through a concerted effort to recruit new and experienced nurses and to establish a basic environment in which neonatal palliative care can be provided.

The final barrier in this study to successful neonatal palliative care was the absence of education for the healthcare team. Although it was not assessed using the tool, only $12.5 \%$ of the nurses and $11.5 \%$ of the physicians received education in neonatal palliative care, and $89.3 \%$ of the nurses and $84.6 \%$ of the physicians mentioned that neonatal palliative care education was necessary. According to Wright et al. [19], 46\% of nurses answered that they had received education in communicating with parents of babies near death and in supportive nursing interventions. They answered unanimously that palliative care was essential in neonatal nursing education. To relieve the pain of neonates who are on the verge of death, support the parents who can do nothing but watch them, and help them cope, it is necessary to develop and implement an educational program on neonatal palliative care for physicians and nurses in the NICU.

This study brought focus to an issue that had received relatively little attention and emphasized the importance of neonatal palliative care. However, there were two limitations in this study. First, it could not fully reflect the cultural characteristics of South Korea as it used a translated tool developed in another country. Second, nurses and physicians have very different characteristics and a direct comparison of their attitudes toward palliative care is difficult in a hierarchical system. Otherwise, the identification of facilitators and barriers for each item in each group can be used as basic data for followup studies on neonatal palliative care.

In this study, Cronbach's alpha, a measure of internal consistency reliability, was 0.63 for "organization" and .64 for "cli- nician," both of which were relatively low. This is considered to be a result of the differences between South Korea and the West in organizational and healthcare team roles. In addition, there were only two items for the clinician category. Based on the results of this study, it is necessary to conduct qualitative research through in-depth discussion of the barriers to neonatal palliative care and to develop a tool that could identify Korea-specific barriers to neonatal palliative care. Lastly, an educational program on neonatal palliative care should be developed and implemented, both for medical professionals and for parents.

\section{CONFLICT OF INTEREST}

No potential conflict of interest relevant to this article was reported.

\section{ORCID}

Ha Na Jung, https://orcid.org/0000-0002-2398-0625

Hyeon Ok Ju, https://orcid.org/0000-0002-0264-2651

\section{AUTHOR'S CONTRIBUTIONS}

Conception or design of the work: HNJ, HOJ. Data collection: HNJ. Data analysis and interpretation: HNJ, HOJ. Drafting the article: HNJ, HOJ. Critical revision of the article: HNJ, HOJ. Final approval of the version to be published: HNJ, HOJ.

\section{SUPPLEMENTARY MATERIALS}

Supplementary materials can be found via https://doi. org/10.14475/jhpc.2021.24.3.165.

\section{REFERENCES}

1. Catlin A, Carter B. Creation of a neonatal end-of-life palliative care protocol. J Perinatol 2002;22:184-95.

2. Lee MC, Chen YC, Chen CH, Lu FL Hsiao CC, Peng NH. Comparison of the educational needs of neonatologists and neonatal nurses regarding palliative care in taiwan. Am J Hosp Palliat Care 2016;33:264-71.

3. Salmani N, Rassouli M, Mandegari Z, Bagheri I, Tafti BF. Palliative care in neonatal intensive care units: Challenges and solutions. IJN 2018;9:33-41. 
4. Statistics Korea [Internet]. 2020 Birth and death statistics. Daejeon: Statistics Korea; 2021 [cited 2021 Feb 24]. Available from: http://kostat. go.kr/portal/korea/kor_nw/1/2/1/index.board? bmode=read\&bSeq=\&aSeq=388265.

5. Wi DH, Kang SJ. Relationship among nurses' knowledge, attitude towards palliative care and perception of death in neonatal intensive care units. Child Health Nurs Res 2016;22:257-64.

6. Statistics Korea [Internet]. 2019 Birth statistics. Daejeon: Statistics Korea; 2021 [cited 2020 Aug 26]. Available from: http://kostat.go.kr/ portal/korea/kor_nw/1/2/3/index.board? bmode=read\&bSeq=\&aSeq=384631.

7. YI YH, Yang HM. Nurse's grief experience and attitudes toward bereavement/ end-of-life care of families of dying Infants in the neonatal intensive care units. JKDAS 2015;17:1045-60.

8. Chen CH, Huang LC, Liu HL, L HY, Wu SY, Chang YC, et al. To explore the neonatal nurses' beliefs and attitudes towards caring for dying neonates in Taiwan. Matern Child Health J 2013;17:1793-801.

9. Peng NH, Chen CH, Liu HL, Lee HY. To explore the conditions of dying infants in NICU in Taiwan. J Crit Care 2012;27:102. e7-13.

10. Quinn, M, Gephart S. Evidence for implementation strategies to provide palliative care in the neonatal intensive care unit. Adv Neonatal Care 2016;16:430-8.

11. Dighe MP, Muckaden MA, Manerkar SA, Duraisamy BP. Is there a role of palliative care in the neonatal intensive care unit in India? Indian J Palliat Care 2011;17:104-7.

12. Mendel TR. The use of neonatal palliative care: Reducing moral distress in NICU nurses. JNN 2014;20:290-3.

13. Parravicini E. Neonatal palliative care. Curr Opin Pediatr 2017;29:135-40.

14. Catlin A, Brandon D, Wool C, Mendes J. Palliative and end-of-life care for newborns and infants: from the national association of neonatal nurses. Adv Neonatal Care 2015;15:239-40.

15. Hamric AB, Blackhall $\amalg$. Nurse-physician perspectives on the care of dying patients in intensive care units: collaboration, moral distress, and ethical climate. Crit Care Med 2007;35:422-9.

16. De Rooy L, Aladangady N, Aidoo E. Palliative care for the newborn in the United Kingdom. Early Hum Dev 2012;88:73-7.

17. Hong CY, Ng GN, Poulose J, Lin NJ, Goh CR. Attitude of doctors in an Asian oncology centre towards referral to palliative care. J Palliat Care 2011:27:157-63.

18. Abdolghani A, Mohammadreza F, Fatemeh A, Nasrollah A. Nurses versus physicians' knowledge, attitude, and performance on care for the family members of dying patients. Korean J Med Educ 2016;28:79-85.

19. Wright V, Prasun MA, Hilgenberg C. Why is end-of-life care delivery sporadic? : A quantitative look at the barriers to and facilitators of providing end-of-life care in the neonatal intensive care unit. Adv Neonatal Care 2011;11:29-36.

20. Kain V, Gardner G, Yates P. Neonatal palliative care attitude scale: development of an instrument to measure the barriers to and facilitators of palliative care in neonatal nursing. Pediatrics 2009;123:2e07-13.

21. Azzizadeh Forouzi M, Banazadeh M, Ahmadi JS, Razban F. Barriers of palliative care in neonatal intensive care units. Am J Hosp Palliat Care 2017;34:205-11.

22. Seol EM, Koh CK. Critical care nurses' attitudes toward hospice and palliative care and their related factors. Perspectives in Nursing Science 2015;12:94-105.

23. Galletta M, Portoghese I, Carta MG, D’Aloja E, Campagna E. The effect of nurse-physician collaboration on job satisfaction, team commitment, and turnover intention in nurses. Res Nurs Health 2016;39:375-85.

24. Kang KA, Kim HS, Kwon SH, Nam MJ, Bang KS, Yu SJ, et al. Nurses' Knowledge of and Attitudes toward Pediatric Palliative Care of Korea. Korean J Hosp Palliat Care 2014;17:289-300.

25. Falck AJ, Moorthy S, Hussey-Gardner B. Perceptions of palliative care in the NICU. Adv Neonatal Care 2016;16:191-200.

26. Yam BM, Rossiter JC, Cheung KY. Caring for dying infants: experiences of neonatal intensive care nurses in Hong Kong. J Clin Nurs 2012;10:651-9.

27. Kim YM, Yoon HY, Choi YJ, Shin DS. Neonatal ICU nurses' coping with death of high risk newborn. J Health Info Stat 2019;44:103-10.

28. MOHW [Internet]. Press release: Neonatal intensive care unit national health insurance fee to improve. Sejong: Ministry of Health and Welfare; 2018 [cited 2018 Apr 24]. Available from: http://www.mohw.go.kr/react/al/sal0301vw.jsp?PAR_MENU_ID=04\&MENU_ ID =0403\&page=92\&CONT_SEQ=344636.

29. Ko BJ, Yu M, Kang JS, Kim DY, Bog JH, Jang EK, et al. A study on identifying nursing activities and standard nursing practice time for developing a neonatal patient classification system in neonatal intensive care unit. J Korean Clin Nurs Res 2012;18:251-63. 